\title{
Span operational aspects under offsetting the axis of the track panel
}

\author{
Alexey Loktev ${ }^{1}, V$ adim Korolev $^{1}$, Irina Shishkina ${ }^{1 *}$, Olga Lokteva ${ }^{1}$, and Ekaterina \\ Gridasova $^{2}$ \\ ${ }^{1}$ Russian University of Transport (MIIT), Chasovaya str. 22/2, Moscow, 125190, Russia \\ ${ }^{2}$ Far Eastern Federal University, Sukhanova St., 8, Vladivostok, 690091, Russia
}

\begin{abstract}
The stress-strain state of a small railway bridge with a beam design is considered in the article for the case of offsetting the track panel axis relative to the bridge axis by the value exceeding the limit determined by regulatory documents. The differential equation is considered in the analytical calculation of the behavior parameters and the state of the span under the action of the load. This equation describes the vertical vibrations of the beam and allows considering them as a combination of forced and free vibrations. In numerical modeling, the finite element method is used as the solution procedure. Determining equations of the method contain linear and angular displacements of nodes in the model as unknowns. As a result of the calculations, graphical dependences for normal and horizontal displacements, internal forces, principal and equivalent stresses at various points of the span are obtained. Values are presented that show an increase in bending and torsional forces, as well as in principal stresses when the axis of the railway track is displaced relative to the bridge axis.
\end{abstract}

\section{Introduction}

Artificial structures form an integral part of the railways, both operated and yet only designed, and in the latter case, bridge crossings and transport tunnels are more common than in historic projects. This is due to the development of construction technologies, the emergence of new structural materials, with more stringent requirements for shrinkage of the upper track structure, as well as to an increase in the rolling stock speed and an increase in axle load and the total weight of trains.

The combined action of the upper structure of the railway track and the supporting bridge crossing structures is an important condition for the normal operation of the railway. It allows section to be operated without various restrictions and additional work on the current maintenance and diagnostics of structural elements. The conceptual model "bridge crossing - the upper structure of the track" or the combined model "bridge crossing - the upper structure of the track - vehicle" were considered in many domestic and foreign studies. Most often, the joint deformation of the track and bridge structures under the action of a moving load was studied, as well as stationary and non-stationary processes in

\footnotetext{
* Corresponding author: shishkinaira@inbox.ru
} 
individual elements or in the entire structure as a whole $[1,2]$. The conducted studies were aimed at optimizing the design of bridge crossings in terms of the span stiffness, crossbar supports and viscoelastic support parts. The goal of the research was also to optimize the mutual influence of the vibrations of stationary structures and vehicle elements under various movement modes of rolling stocks and various types of movable load in them [3, 4].

The most modern approach used in the calculation of bridge crossings under a moving load involves a study based on the model "bridge crossing - the upper structure of the track - vehicle". In this case, the operational aspects of structural elements for various modes of rolling stock movement, geometric and mechanical parameters of the span structure are revealed. The characteristics of the transported cargo, the presence and development degree of defects in running surfaces, etc., are also determined. It is also necessary to take into account the probabilistic nature in the interaction of vehicles and a bridge structure. This is due to the occurrence of vertical and horizontal inertia forces, the time dependence of the average dynamic characteristics, the change in the case of uneven inertia motion, the center of gravity position of the stock, the geometric position effect and also the presence of rail defects lashes on the frequency of kinematic disturbance, varying along the length of the span.

Currently, the dynamic models of uneven movement, which considering the abovementioned factors, are not always used in the field of constructing the transport infrastructure at the level of solving engineering problems. Thus, simplified approaches are still implemented. For example, the equally slow braking of rolling stocks having the same workload is considered. The load transmitted on the bridge structure was modeled by concentrated forces applied to predetermined points of the span.

\section{Research technique}

The modern transport system involves an increase in the length of roads and in the intensity of their operation. At the same time, there is an increase in the load carrying capacity of artificial structures and, often, a deterioration in their technical maintenance during operation. The accurate assessment of the condition and operational reliability of existing artificial structures can be singled out as a separate task, which will be further considered. The considered structures are not only located on the main highways, but also on the approach roads of industrial enterprises (Fig. 1) [5, 6]. The scheme and cross section of the span bridge crossing, which is located on the site of a single-track non-electrified railway, are shown in Fig. 2.

An essential aspect in solving engineering problems regarding the design of construction and reconstruction of transport infrastructure facilities is the need to dampen forced vibrations of elements of artificial structures. It requires providing viscoelastic support parts that can reduce resonance phenomena, control the amplitude of the building's vibrations at design loads. Rubber-metal supporting parts are often used for this purpose, they are installed in the location where the span rests on the supporting structure. As the main parameters determining the bridge crossing operation, geometric (displacement), kinematic (acceleration during vibrations) and dynamic (forces acting in various elements of the bridge according to the applied load) characteristics can be used [7, 8]. The parameters of viscous resistance, stiffness coefficient of the component elements and the entire structure as a whole are also important characteristics that determine the operation of the artificial structure.

The values of the exposure periods of the sprung weight in the crew are important when taking into account the movement of the vehicle along the bridge. Usually their values are in the range $0.5-1.0 \mathrm{~s}$. For bridges with small spans, as presented in Fig. 1, 2, a slight 
effect from vehicle vibrations is common. This is explained by the short duration of the dynamic impact of the rolling stock and the artificial structure, as well as by the fact that the amplitude of the span vibrations is small compared to the vertical movements of the vehicle. The absence of feedback effect increases the margin for the first and second group of limiting states for the structure as a whole $[9,10]$. In world engineering practice, cases of oscillations are known regarding bridge structures with significant amplitudes of vertical movements during passage with low speeds of long trains with a large axle load. However, since the considered road section connects an industrial enterprise with a section of the III category track, such phenomena won't be taken into account in the study.

Let us consider a small reinforced concrete railway bridge, the total length of which is $18.80 \mathrm{~m}$, the length of the main bridge span is $7.80 \mathrm{~m}$, the opening of the bridge is $6.50 \mathrm{~m}$ (Fig. 2a). The bridge is on a straight section of the track, there is no slope, the bridge was built in 1955. Two ribbed reinforced concrete beams with a height of $1.50 \mathrm{~m}$ are used as a span. The distance between the axles is $2.90 \mathrm{~m}$, the sides of the span are increased by 0.30 $\mathrm{m}$, the height of the ballast layer is $0.60 \mathrm{~m}$, the width of the span is $4.20 \mathrm{~m}$ (Fig. 2b).

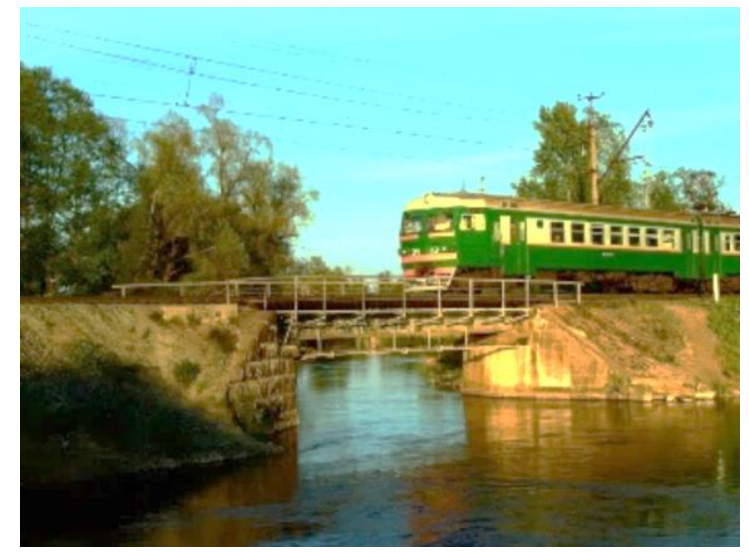

Fig. 1. General view of the bridge

During the regular inspection of the artificial structure, the deviation of the track panel axis from the span axis was 15 centimeters, along with some other deviations from the design position and defects in the structural elements. Such deviation exceeds the standard values on Russian railways for straight sections of the track in three times.

Let us consider the changes in operation and in the parameters of the stress-strain state of the bridge crossing, when the axis of the track panel is displaced relative to the axis of the bridge by an amount greater than the normative allowable.

The design of the main span is presented in the form of a traditional single-span hinged beam (Fig. 2a). Let us adopt several major assumptions. Firstly, the considered oscillations are small comparing to the geometric dimensions of the bridge and its individual elements (the beam system is linearly deformable). Secondly, the material obeys Hooke's law. Thirdly, the inertia of rotation during low-frequency oscillations are not taken into account; the mass of the upper track structure is included in the masses of the corresponding bridge structures and is taken to be uniformly distributed. Fourthly, rubber-metal bearing parts are modeled by ideal hinges, support devices and joints are also ideal. After all, the internal friction of the span elements is described by the Voigt model [11, 12, 13]. 
a)
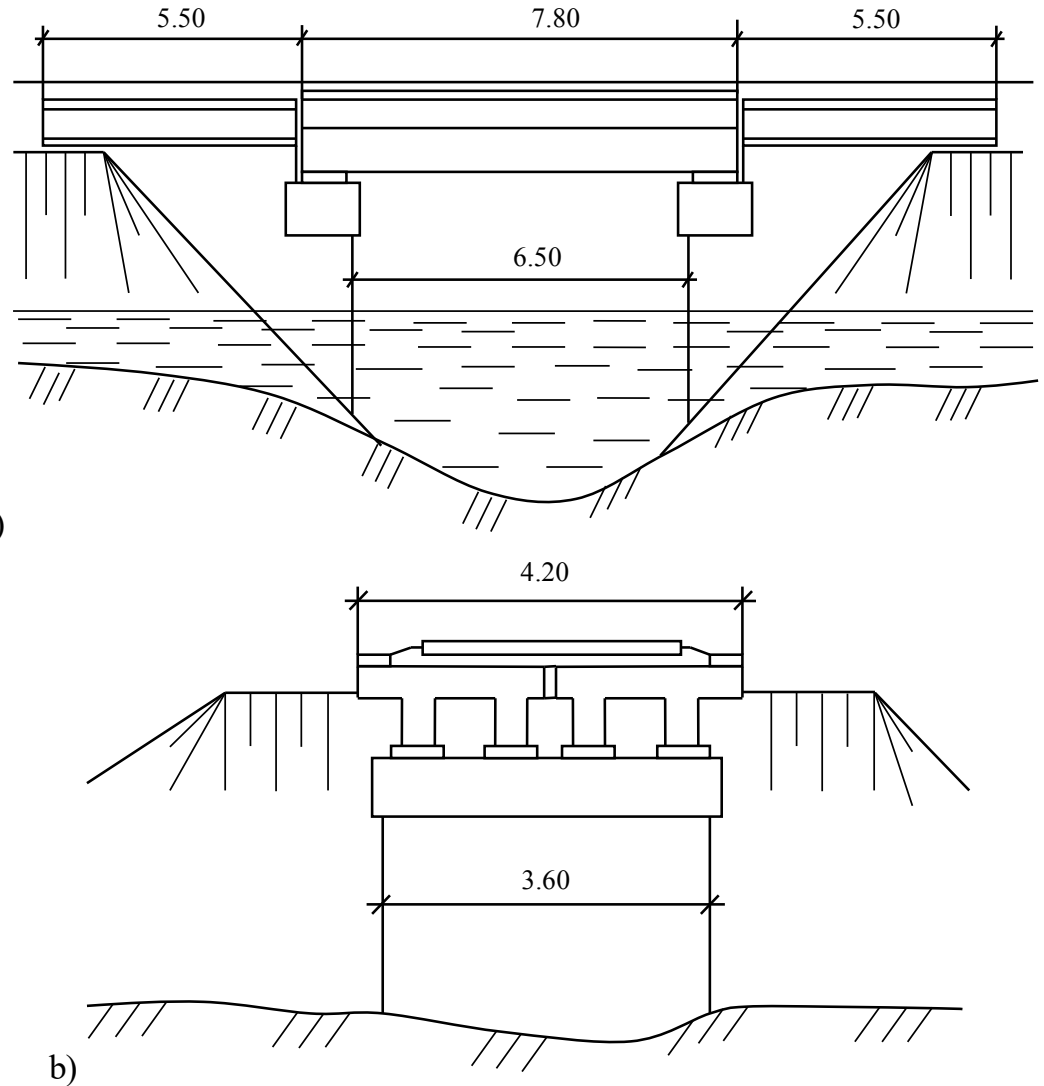

Fig. 2. The scheme of the bridge: a) facade, b) cross section along the axis

The differential equation describing the vertical vibrations of the beam and allowing considering them as a combination of forced and free vibrations can be represented as follows:

$$
m_{b} \frac{\partial^{2} w(x, t)}{\partial t^{2}}+E I_{z}\left(1+\mu_{0} \frac{\partial w(x, t)}{\partial t}\right) \frac{\partial^{4} w(x, t)}{\partial x^{4}}=\sum_{i=1}^{n} P_{i}(t) \delta\left(x-\gamma_{i}\right)\left[\eta\left(t-t_{0}\right)-\eta\left(t-t_{1}\right)\right],
$$

here $w$ is the vertical displacement of the midline of the beam, $m_{b}$ is the linear mass of the beam. $E I_{z}$ is the beam stiffness during bending in the vertical direction, $\mu_{0}$ is the reduced coefficient of internal friction. $P_{i}(t)$ is the dynamic pressure from the $i$-th axle of the vehicle, taking into account the eccentricity with respect to beam axis, $n$ is the number of vehicle axles that can simultaneously fit on the main span of the bridge. $\delta()$ is the Dirac delta function, $\gamma_{i}$ is the time moment when the $i$-th rolling stock axle approaches a given point of the span, $\eta$ ( ) is the unit function showing the instant of occurrence of $t_{0}$ and $t_{1}$ when the moving load is departure on the bridge.

The expression for the vertical displacements of the wheelset should be added to the differential equation describing the dynamic behavior of the span beam, taking into account the interaction force between the wheel and rail $[11,12]$ :

$$
m_{k i} \frac{\partial^{2} y(x, t)}{\partial t^{2}}=-P_{i}(t)
$$

here $m_{k}$ is the rolling stock mass reduced to the $i$-th wheel pair, $y$ is the vertical movement of the wheel.

The relations, which connect the vertical displacements of various elements in the oscillatory system with resolving the issue of contact interaction of the wheel and rail, should be added to equations (1) and (2): 


$$
\begin{gathered}
y=\alpha+w\left(\gamma_{i}, t\right), \\
\alpha=k^{\prime} P^{n^{\prime}},
\end{gathered}
$$

here $\alpha$ is the local deformation in the sector of interaction between the wheel pair and the rail, $k^{\prime}$ is the coefficient depending on the geometric and mechanical properties of the contacting bodies, as well as the materials of the structural elements directly under the contact area.

In order to solve functional equations (1) and (2), it is necessary to take into account the initial conditions:

$$
y\left(t=t_{i}\right)=0, \frac{\partial y\left(t=t_{i}\right)}{\partial t}=V_{0}, w\left(x, t_{i}\right)=0, \frac{\partial w\left(t=t_{i}\right)}{\partial t}=0,
$$

where $V_{0}$ is the vertical component of the rolling stock speed when a wheelset hits a given point of the rail bar.

Using the equations (1), (2), and taking into account relations (3), (4) and initial conditions (5), the determining integro-differential equation with respect to one of the vertical displacements of the oscillatory system can be obtained. It is proposed to search for the numerical solution of this equation by using a computer, so the oscillation period was divided into $k$ parts. The duration of the elementary time interval $\tau$ depends on the frequency of natural vibrations of the span structure and the propagation velocity of wave fronts, in the case when non-stationary wave processes are considered [13, 14]. With a sufficiently small division step of the characteristic time period, it can be assumed that, at each elementary interval $(k-1) \tau \leq t \leq k \tau$, the unknown functions determining the dynamic behavior of the bridge crossing linearly depend on time. Thus, the first derivative of an unknown function can be calculated as the ratio:

$$
\frac{\partial f(k \tau)}{\partial t}=\frac{\left(f_{k}-f_{k-1}\right)}{\tau}
$$

where $f$ is an unknown function of time determined in the solution process.

Higher order derivatives of the sought-for functions are determined in accordance with the same ideology, using templates with large number of support points similar to those used in finite-difference computational schemes [15, 16].

The recurrence expression can be obtained by substituting this relation into the defining equation, which will allow determining the value of unknown function at each moment in time $\tau_{i}=\tau i$.

The solution of the compiled finite-difference equations converges to the exact solution of the boundary value problem with a decrease in the integration interval. The proposed calculating scheme may use grid patterns with a constant partition interval (regular grid patterns) or the integration step in a region with a significant gradient of the desired function may decrease (adaptive grid patterns).

A feature of the presented iterative procedure is that in order to achieve a high solution convergence rate, approximation errors of differential equations of initial and boundary conditions must have the same order of values. In this case, the effect of an increase in errors is observed with a significant decrease in the integration interval. It is associated with the implementation of rounding algorithms in the computer environment and the appearance of errors and inaccuracies associated with dividing by a value close to zero.

The described procedure can be automated both by using the existing mathematical computing packages MathCAD, MATLAB, Maple, Mathematica, and by using the capabilities of the programming languages $\mathrm{C}++$, Delphi, $\mathrm{C} \#$ and the corresponding traditional libraries. The program code written and implemented in the $\mathrm{C}++$ language environment was implemented in the research.

The correlations between the vertical displacements of the midpoint of the median in case when the axis of the track panel coincides with the axis of the bridge crossing (solid 
line) are presented in Fig. 3. Median line connects the gravity centers of the cross sections of the span. The case when the axis of the track panel is displaced $15 \mathrm{~cm}$ to the right of the bridge axis (dashed line) is presented in Fig. $2 b$.

When plotting graphical dependencies in Fig. 3, the estimated length of the span was taken equal to $7.40 \mathrm{~m}$, which corresponds to the calculation scheme in Fig. 2a and the existing support elements. The calculation defining the equations was carried out at a rolling stock speed of $30 \mathrm{~km} / \mathrm{h}$, the 18-9890 freight gondola car was loaded with an axle load of 20 tons and a base of $1850 \mathrm{~mm}$. The dimensions of the main span of the bridge crossing allow considering the movement of only one such carriage (with two wheelsets). When analyzing the obtained graphs, one can note an increase in the amplitude of vertical displacements of the selected span point. This occurs in the presence of a displacement of the track panel axis relative to the bridge axis and a certain time shift of the extrema of the deflection function.

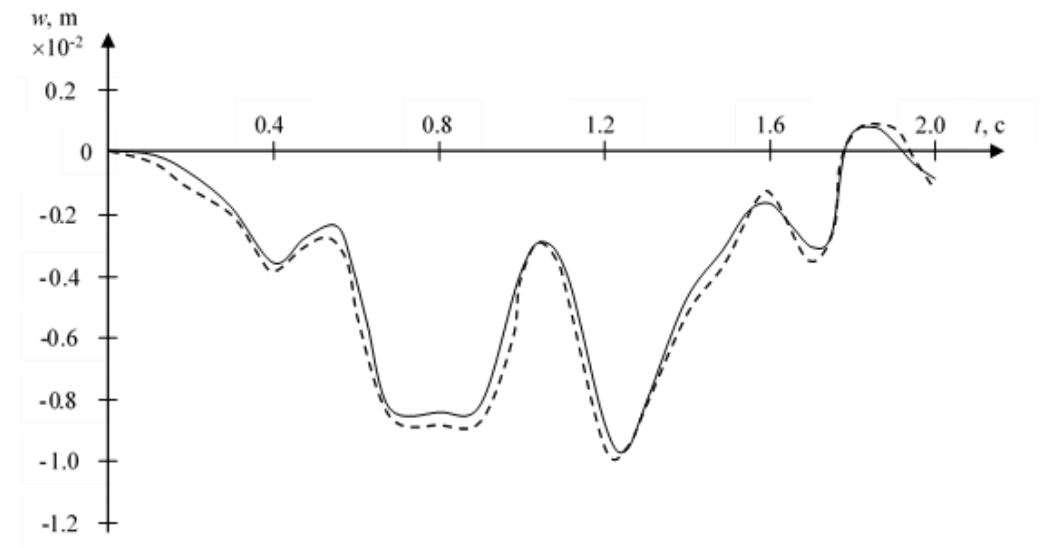

Fig. 3. The correlation between the vertical displacement of the midline of the span beam and time at a point with coordinate $x=l / 2$

The deformation processes in spans of bridge crossings, in case of vehicles moving along them, are quite complex and must take into account many factors related to the structures of the construction. The movement of vehicles, the nature of the goods transported, weather conditions and other reasons should be considered. An attempt to solve such a problem is analytically connected with computational difficulties. Moreover, it is also connected with the need for using the additional conditions that ultimately limit the application scope of the obtained solutions, and does not provide sufficiently simple final expressions, which could be used in engineering practice $[18,19]$. The analytical approach described above allows calculating the characteristics of the deformed state at individual points of the bridge structure under specified influences. In order to determine the operation nature of the entire span, it is proposed to use computer digital simulation methods. The finite element method was used as the main numerical method for calculating the bridge span. The defining equations of this method contain linear and angular displacements of nodes of the model as unknowns. This method and its various modifications are widely used in modern computing software systems, including those that have passed the state licensing and verification procedure for various engineering tasks, types of structures and structures.

The construction of the considered span is presented in the as a set of finite elements of a standard type (rods, plates, shells, bonds with finite stiffness, one-way bonds, etc.), interconnected in nodes. The type of finite element used depends on the geometric (shape, fixing method, etc.), mechanical (the law of element deformation, the correlation between 
the displacements of the nodes of an individual element and the nodes of the model as a whole) characteristics. Furthermore, it depends on the type of material and stiffness parameters when working at a given load in the compiled model [19, 20].

An important element of the structure model is a node that is modeled by a rigid infinitesimal body. Its position in space is determined by three linear $(U, V, W$ - linear displacements along the $x, y$ and $z$ axes) and three angular $(\alpha=\partial w / \partial y, \beta=-\partial w / \partial x, \gamma-$ rotation angles relative to the $x, y$ and $z$ axes, respectively) displacements. The system of canonical equations of the displacement method for finite elements is formed by imposing additional constraints on all possible displacements and equating the forces arising in these constraints to zero. This assumption allows forming a system, whose number of equations is equal to the number of unknown displacements, which are then determined digitally in the software package $[21,22]$.

In the nodes where the span rests on the support beam, there are ties restricting the movement of the components of the finite element model of the span beams. This corresponds to the presence of rubber-metal bearing parts and rests preventing horizontal displacements at the points of support.

In order to compose the equilibrium equations determining the deformation of an individual finite element and their combination, it is proposed to use the principle of possible displacements. According to this principle, each element point can receive an additional small displacement $-\delta u$, and the main displacements of the points of the deformable element are represented by the $\bar{u}$ vector. After equating the increment of the work of internal forces $\delta U$ at possible displacements of the body to the increment of work of external forces $\delta W$, presented as the sum of the work of the load $q$ distributed over the volume $V$ and the load $p$ distributed over the surface $\mathrm{S}$, the main functional relation can be represented in the following form

$$
\int_{V} \sigma \cdot \delta \varepsilon \cdot d V=\int_{V} \bar{q} \cdot \delta \bar{u} \cdot d V+\int_{S} \bar{p} \cdot \delta \bar{u} \cdot d S
$$

where $\sigma=\sigma_{i j} \bar{e}_{i} \bar{e}_{j}$ is the stress tensor, $\varepsilon=\varepsilon_{i j} \bar{e}_{i} \bar{e}_{j}$ is the strain tensor, $\bar{e}_{i}$ and $\bar{e}_{j}$ are the unit orthogonal vectors, $i, j$ take values $1,2,3$.

Based on the assumption that the deformations of the elements are small, the relation can be written down, the right side of which is a tensor

$$
\varepsilon=\nabla \bar{u}=\frac{1}{2}\left(\frac{\partial u_{i}}{\partial x_{j}}+\frac{\partial u_{j}}{\partial x_{i}}\right) \bar{e}_{i} \bar{e}_{j}
$$

where $x 1, x 2, x 3$ are the coordinate axes corresponding to the direction of unit vectors. The stress tensor and strain tensor for a finite element with the elastic properties of the material are connected by the relation:

$$
\sigma_{i j}=D_{i j k l} \varepsilon_{k l},
$$

where $D_{i j k l}$ is tensor operator defining the elastic mechanical properties of an element. Given the expressions (8) and (9), the defining equation (7) can be written in the following form

$$
\int_{V} D \bar{V} \bar{u} \cdot \delta(\nabla \bar{u}) \cdot d V=\int_{V} \bar{q} \cdot \delta \bar{u} \cdot d V+\int_{S} \bar{p} \cdot \delta \bar{u} \cdot d S
$$

Equation (10) can be represented as matrix in order to describe the equilibrium of an individual finite element, if the replacement $\delta \bar{u}=\Phi_{i} \cdot \delta \lambda_{i}$ is used

$$
\{K\}\{\lambda\}=\{f\}
$$


where $\{K\}=K_{i j}=\int_{V} \nabla \Phi_{i} \cdot D \cdot \nabla \Phi_{j} \cdot \lambda_{i} d V$

is a matrix that sets the stiffness parameters of $\{f\}=f_{i}=\int_{V} \bar{q} \cdot \Phi_{i} \cdot d V-\int_{S} \bar{p} \cdot \Phi_{i} \cdot d S$

the element in different directions,

is the load vector acting at the nodes of the element in the direction of the main unit vectors, $\{\lambda\}=\lambda_{i}$ is the displacement vector of the nodes of the design scheme, $i, j=1,2, \ldots N$.

In order to take into account the dynamic behavior of structures, it is necessary to obtain the following equations of motion on the basis of the equilibrium equations (11) and the use of the d'Alembert principle, which allows representing volume forces of inertia

where $\{M\}=M_{i j}=\int_{V} \rho \cdot \Phi_{i} \cdot \Phi_{j} \cdot d V$

$$
\{M\}\{\lambda\}+\{K\}\{\lambda\}=\{f\}
$$

is matrix of masses, $\rho$ is density of element's

material, $\rho \cdot \Phi_{j} \cdot \gamma_{j}=\rho \frac{\partial^{2} \bar{u}}{\partial t^{2}}$ are the volumetric inertia forces.

The solution to the system of equation (12) is the displacement vector of the nodes in the model, through which then the displacements of the remaining points of the elements and the entire structure can be determined, as well as strain and stress [23].

The functions describing the form of displacements of points of various finite elements in the implemented finite element method are represented approximately using simplified dependencies. The dependences used affect the indicator $k$ included in the expression for estimating the resulting error when determining displacements, deformations, forces, and stresses. The expression depends on the ratio $(h / L)^{k}, h$ is the maximum step of numerical integration. $L$ is the characteristic geometric size of the fragment under consideration.

The model of the span is proposed to be composed of five types of finite elements:

1) The elastic bond of finite stiffness, which is used to simulate the resting of span beams on the support beams and actually represents the compressive or shear stiffness reduced to a single linear or angular displacement, respectively. The first and second bed coefficients for this element existing in the software package are multiplied by the surface area of the element interacting with the support unit.

2) An axial element, the displacements of points of which are modeled by third degree polynomials and transcendental expressions, depending on the absence or presence of shear deformations. Such an element is deformed under the influence of the longitudinal force $N$, bending moments $M_{y}, M_{z}$, the corresponding transverse forces $Q_{z}$ and $Q_{y}$, and torque $M_{k}$.

3) A flat element with four nodal points in the plan, the normal displacements of which are modeled by an incomplete polynomial of the fifth degree. At each nodal point there is a vertical displacement $w$; rotation angle around the $x$ axis $\alpha=\partial w / \partial y$; rotation angle around the $y$ axis $\beta=-\partial w / \partial x$. Such a finite element is used for calculating thin plates, it determines bending moments $M_{x}, M_{y}$, torque $M_{x y}$, shear forces $Q_{x}, Q_{y}$, as well as the reactions of supports in the attachment points with adjacent elements.

4) A shell element with three nodal points, on a small surface area its shape can be represented by a flat figure. The normal displacements of the points of such an element are represented by a polynomial of the fourth degree, and the tangent displacements are represented by a polynomial of the first degree.

5) A shell element with four nodal points, the normal displacements of the points of such an element are represented by a polynomial of the third degree, and tangent displacements are modeled by an incomplete polynomial of the second degree.

In general, it can be noted that linear displacements $U, V$ correspond to membrane 
deformations. Linear displacements $W$ and angular displacements $\alpha, \beta$ correspond to bending deformations. Angular displacement $\gamma$ is used to connect elements that do not lie in the same plane and is responsible for the spatial deformation of the set of finite elements in the local coordinate system for each element $\gamma=0$.

The model of the bridge span in the form of a system of finite elements with their numbers is shown in Fig. 4.

The appearance of the structure in simulation mode is shown in Fig. 5, it corresponds to the passport data of the bridge crossing and the results of field measurements (Fig. 2).

When calculating the stress-strain state of the span, normative static and dynamic loads and their combinations were taken into account. The dynamic behavior of the model was taken into account by using decomposition into the forms of natural vibrations, the first six forms were taken into account.

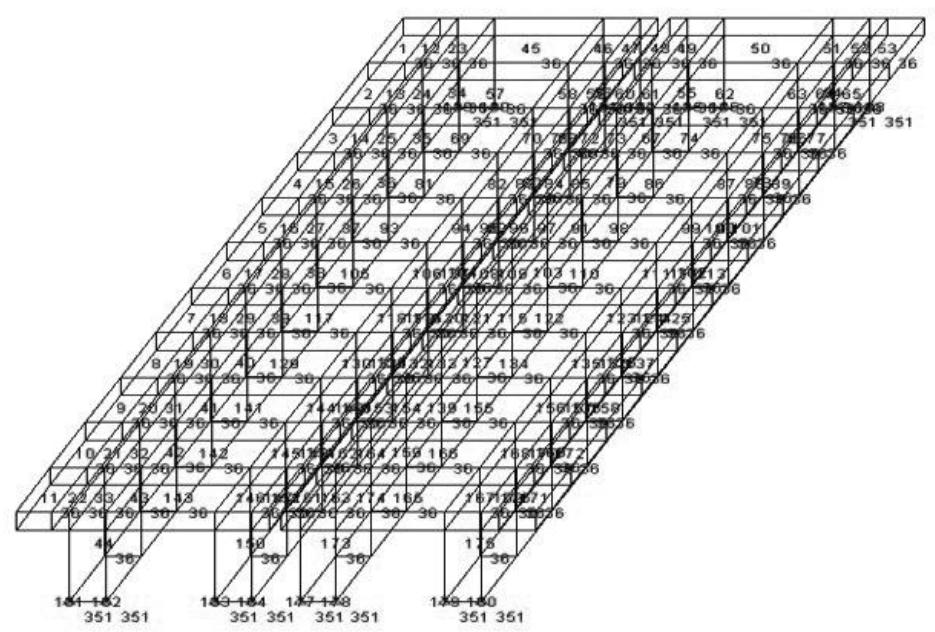

Fig. 4. The model of the span with the numbers of finite elements

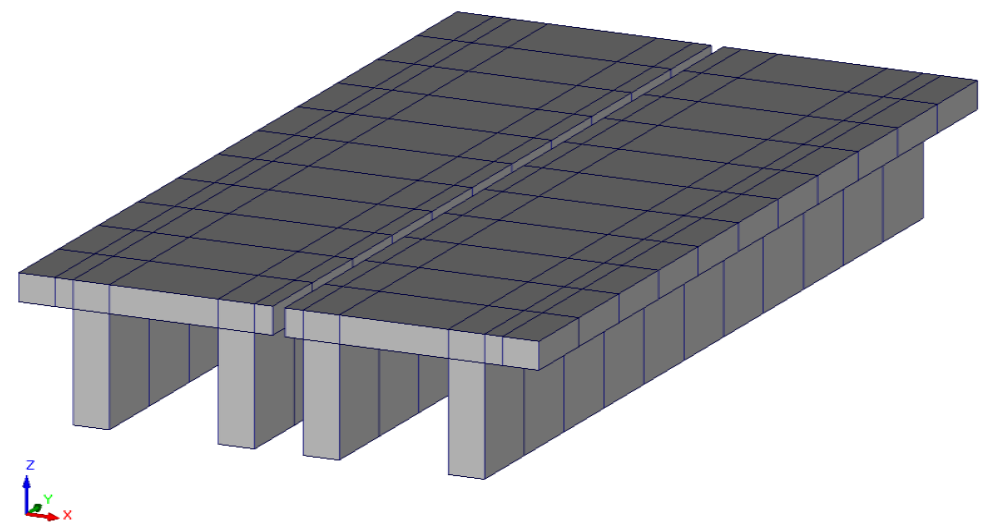

Fig. 5. The appearance of the model of the span

Additional unknowns that are not node displacements can be used when calculating the basic unknowns of the system of determinative equations (12). This is for considering a number of factors associated with dynamic processes and certain types of deformation. For example, these unknowns can be the second mixed derivative of the surface function of a flat element during its deformation, the components of the transverse shear and crimping in layered shell elements. These values are auxiliary in nature and are needed to increase the 
accuracy of computational processes. The horizontal displacements of the model's nodes in the direction along the tie bars of the track panel of the upper track structure are shown in Fig. 6. These are the most important displacements when the axis of the railway track is displaced relative to the bridge crossing axis.

After solving the system of equations (12) regarding the displacements of the nodes of the calculated finite element span model, other functions can be determined using the expressions (8) - (11). These functions characterize the behavior and condition of the span when it deforms under the action of given loads and their combinations. For the flat elements forming the basis of the model of the structure under consideration, when visualizing the stress-strain state and presenting the graphical model of the structure under load, displacements, internal forces and stresses at the nodes and in the center of gravity of each elementary element are calculated. Fig. 7, 8 show iso-fields and contours of longitudinal forces along the coordinate axes $x, z$, respectively. The obtained values are most conveniently used for comparison with analytical calculations of beam deformation before and after displacement of the track panel axis relative to the bridge axis.

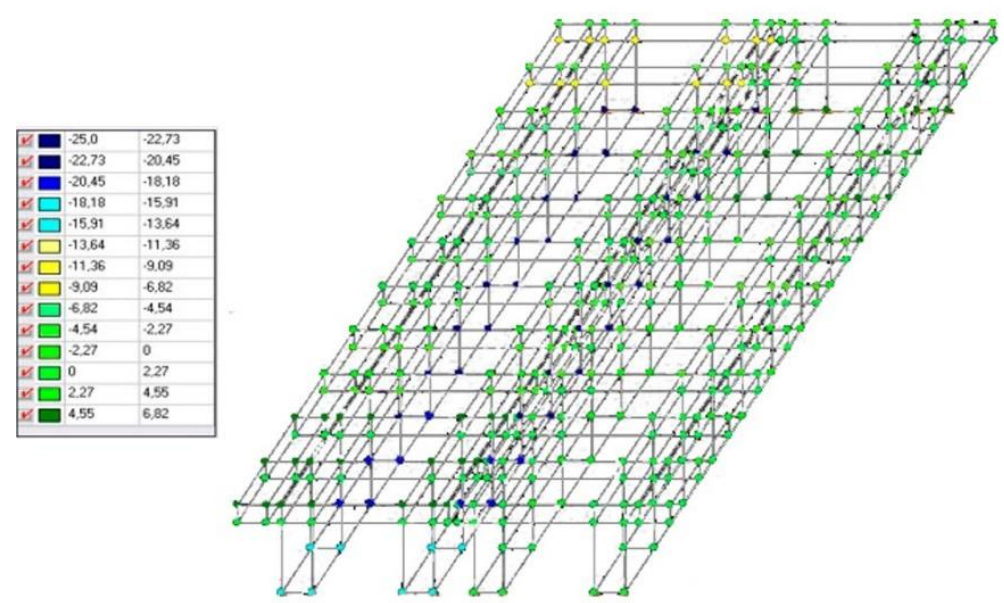

Fig. 6. Horizontal movements of the main nodes in the finite element design scheme from the action of the total load, mm

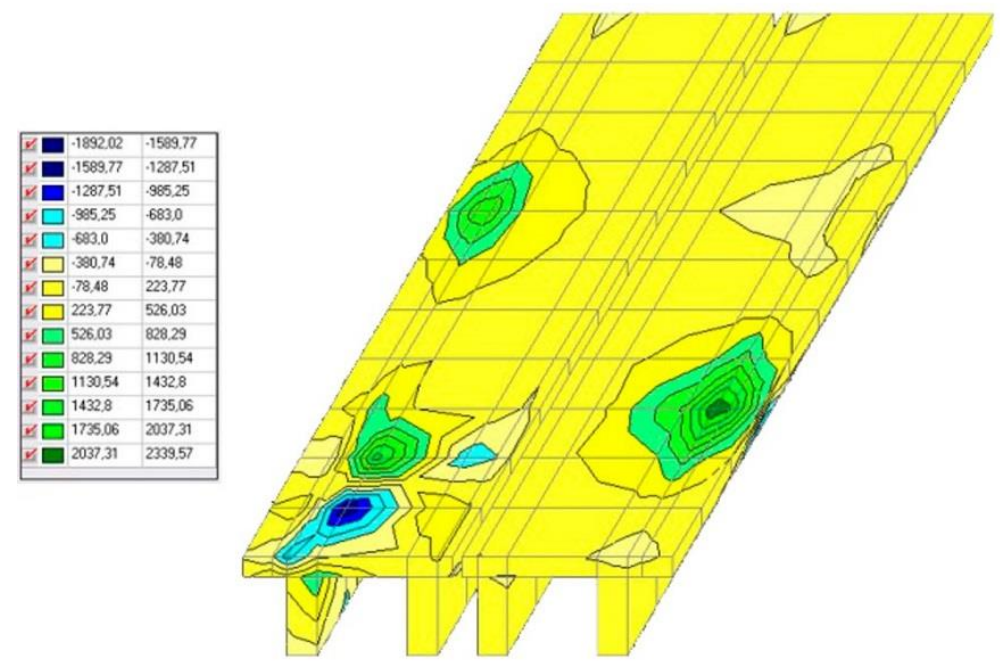

Fig. 7. Longitudinal forces acting along the $x$ axis, $10^{-1} \mathrm{~T} / \mathrm{m}^{2}$ 


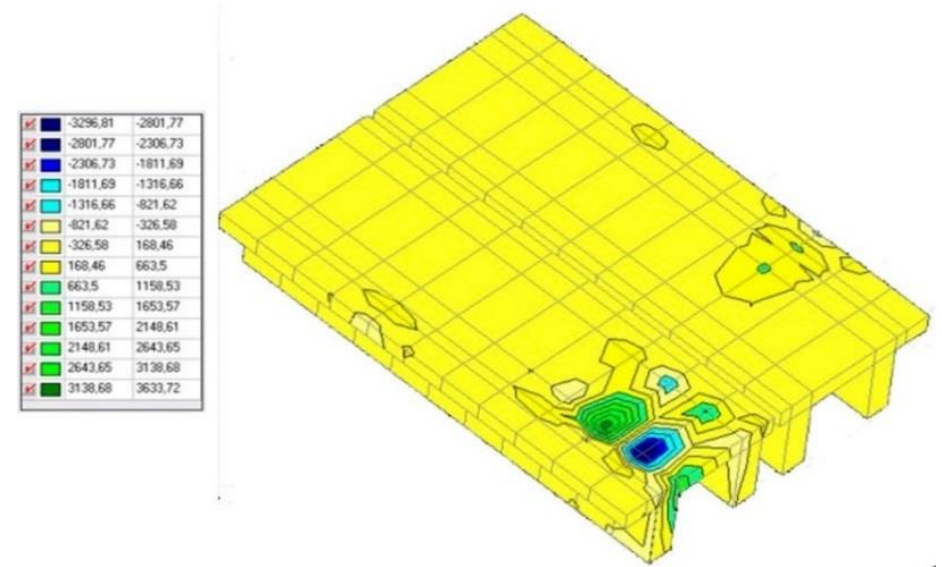

Fig. 8. Longitudinal forces acting along the $z$ axis, $10^{-1} \mathrm{~T} / \mathrm{m}^{2}$

Shear forces, bending moments, torques and transverse forces were also calculated, but their values were included in the functions of tangential and normal stresses, a graphical interpretation of which is presented below. In general, the results presented in Fig. 7, 8 show the most loaded zones of the span, in case when an open railroad freight car 18-9890 is moving along it at a speed of $30 \mathrm{~km} / \mathrm{h}$ with an axle load of 20 tons and a base of 1850 $\mathrm{mm}$, as well as stress concentrators.

The superposition principle is implemented for studying the behavior of a structure with unfavorable combinations of design loads, since it is assumed that structural elements work in the elastic zone of deformations. The maximum of the reduced value is taken as the main criterion for selecting load combinations from possible ones. This value depends on all components of the stress-strain state of a particular finite element [2, 3, 4, 5, 15].

For core finite elements, the main criteria are extreme values of normal and tangential stresses at the corner points of the cross section and the corner points of the core section located on the faces of the cross section.

For shell elements in a plane stress state, the lines enveloping the extrema of normal and tangential stresses are used as determining criteria:

$$
\begin{aligned}
& \sigma(\alpha)=N_{x} \cos ^{2} \alpha+N_{z} \sin ^{2} \alpha+T_{x z} \sin 2 \alpha \\
& \tau(\alpha)=\frac{1}{2}\left(N_{z}-N_{x}\right) \sin 2 \alpha+T_{x z} \cos 2 \alpha
\end{aligned}
$$

For slab elements, determining criteria are calculated by analyzing envelope curves for extreme bending moments and torques:

$$
\begin{aligned}
& M(\alpha)=M_{x} \cos ^{2} \alpha+M_{y} \sin ^{2} \alpha+M_{x y} \sin 2 \alpha \\
& M_{k}(\alpha)=\frac{1}{2}\left(M_{y}-M_{x}\right) \sin 2 \alpha+M_{x y} \cos 2 \alpha
\end{aligned}
$$

In order to simultaneously take into account a greater number of factors affecting the stress-strain state of the span, the main and equivalent stresses at the characteristic points of the finite elements are determined. Representing the stresses as isofields and isolines allows seeing the nature of their distribution over the structure, as well as visually presenting the operation of the entire span as a whole.

The determination of principal and equivalent stresses is based on the assumption that, at any location near the characteristic point of the element, the normal $v$ to which has direction cosines $l, m, n$, the conditions for the resulting voltage $S_{v} \leq \sigma_{1}$ and $S_{v} \geq \sigma_{3}$ are 
satisfied. Here $S_{v}$ is the resulting voltage for normal $\sigma_{v}$ and tangent $\tau_{v}$ stress, $\sigma_{1}, \sigma_{2}$ and $\sigma_{3}$ are the principal stresses $\left(\sigma_{1} \geq \sigma_{2} \geq \sigma_{3}\right)$.

Since for flat elements the characteristics of the stress-strain state differ on the lower, upper, and median surfaces, it is proposed to determine the principal and equivalent stresses at three points of the cross section. Since the span is in a complex stress state, it is necessary to use strength theories that allow comparing the equivalent stress $\sigma_{e}$ with a certain limiting value, which corresponds to simple uniaxial tension $\left(\sigma_{0}^{+}\right)$or compression $\left(\sigma_{0}{ }^{-}\right)$.

\section{Results and discussion}

The results of numerical modeling and calculations of the main and equivalent stresses in the software package are shown in Fig. 9-13 in the form of isofields and isolines. Fig. 9 shows the distribution of shear stresses in the horizontal plane over the span. Fig. 10 and 11 represent the distribution of the principal stresses $\sigma_{1}$ and $\sigma_{3}$ on the inner face of flat finite elements over the entire beam span. Fig. 12 and 13 represent the distribution of flat finite elements of equivalent stresses over the span on the inner face, which are calculated according to the first and second strength theory, respectively.

The presented graphic results of digital modeling allow estimating the location of the most loaded span zones, when the vehicle is moving with an offset of the track panel axis relative to the bridge axis. When conducting straightening work, it is possible to achieve such an arrangement of the track panel, in which the concentration places of extreme stresses will fall upon the stiffeners of the U-shaped beams of the span. The obtained results of numerical calculations will allow planning work more accurately during the repair of the bridge, strengthen existing reinforced concrete elements or provide additional reinforcement.

The presented distribution of internal forces and stresses allows accurately representing the distribution of power factors and the nature of the operation of the entire span. It can be noted that the exposed stress values in the stretched and compressed zones lie within acceptable limits for reinforced concrete. Their distribution along the length and width of the span generally shows the sufficient uniformity of loading of the beams, despite the displacement of the track panel axis relative to the axis of the bridge crossing. The methodology used and the obtained values of forces and stresses can be used by designers when planning repairs of a railway track on a section containing small bridges with a beam design scheme.

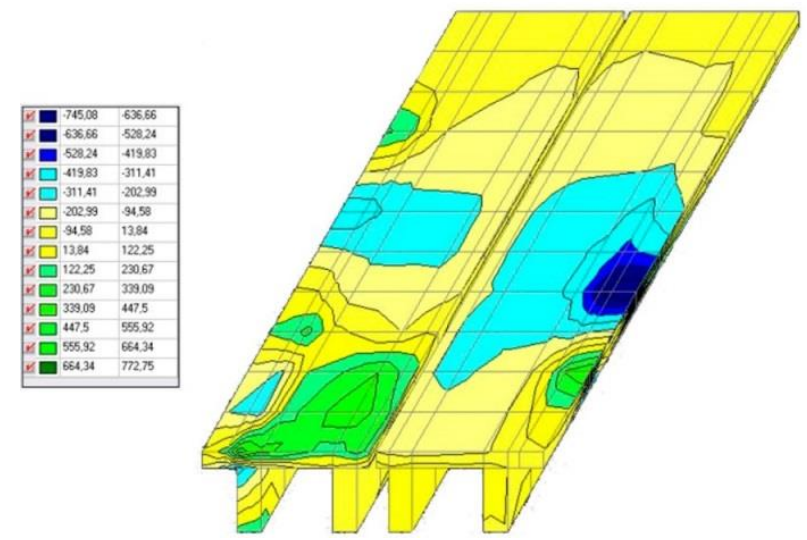

Fig. 9. Shear stresses in the $x y$ plane, $10^{-1} \mathrm{~T} / \mathrm{m}^{2}$ 


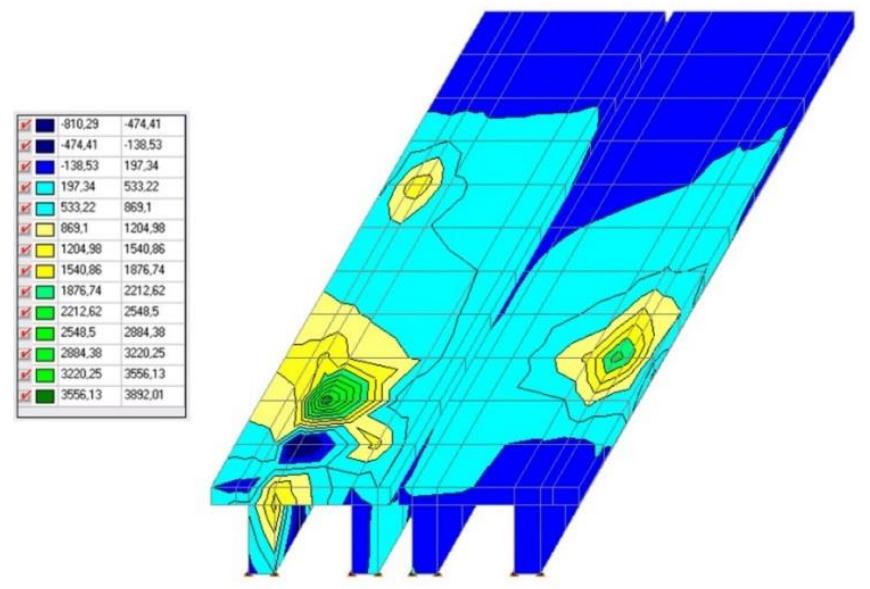

Fig. 10. The main stresses $\sigma, 10^{-1} \mathrm{~T} / \mathrm{m}^{2}$

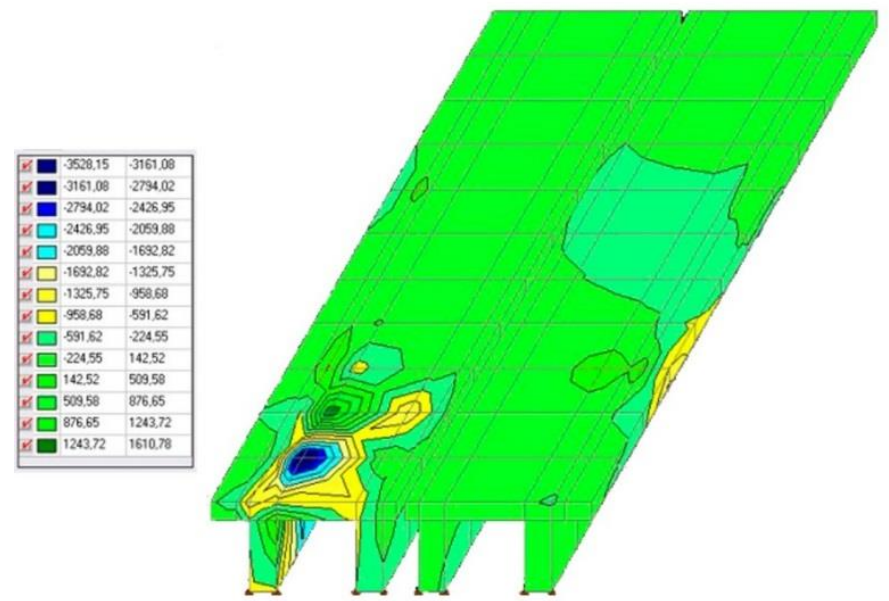

Fig. 11. The main stresses $\sigma_{3}, 10^{-1} \mathrm{~T} / \mathrm{m}^{2}$

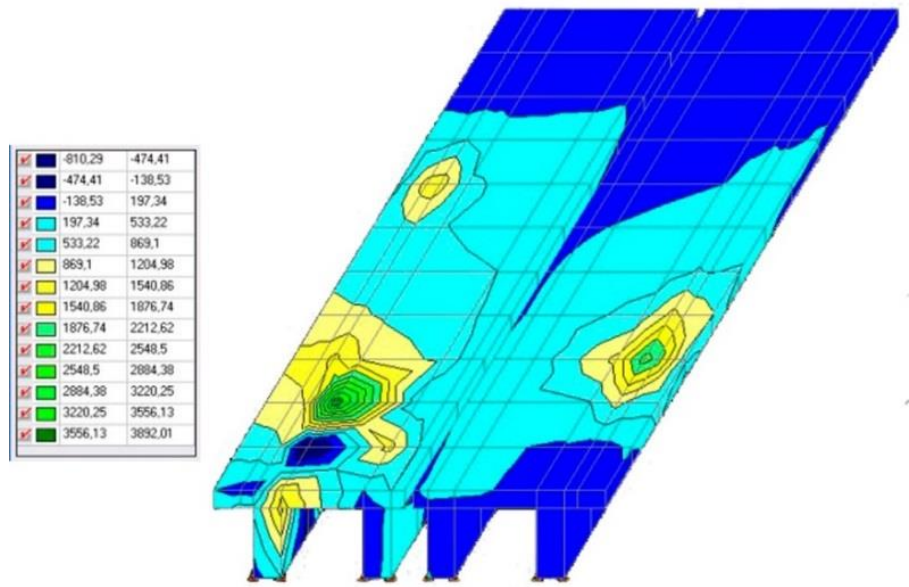

Fig. 12. Equivalent stresses calculated according to the first strength theory, $10^{-1} \mathrm{~T} / \mathrm{m}^{2}$ 


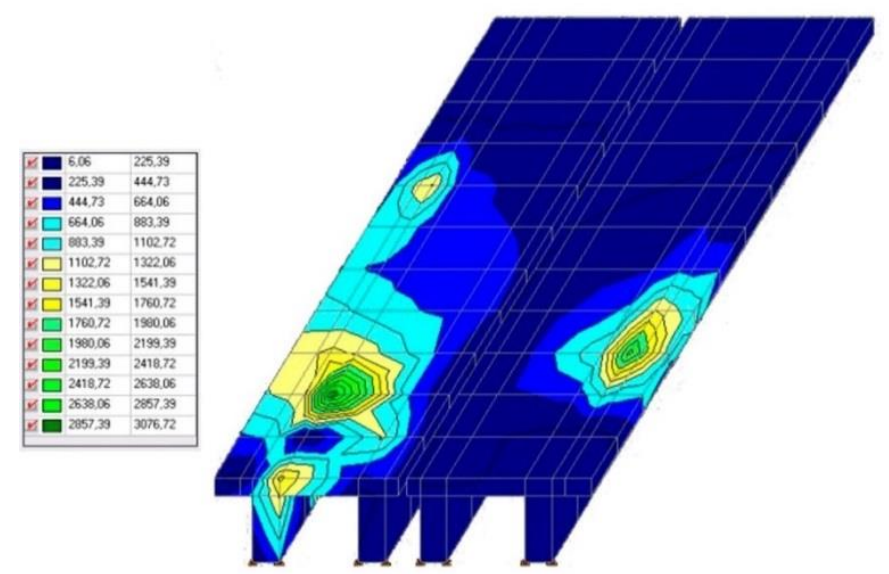

Fig. 13. Equivalent stresses calculated according to the second strength theory, $10^{-1} \mathrm{~T} / \mathrm{m}^{2}$

\section{Conclusions}

Conducted analytical and numerical studies showed the following:

1) The maximum bending moment in one of the span beams when passing a car with an axle load of 20 tons is $710 \mathrm{kN} \cdot \mathrm{m}$ in case of no displacement of the track panel relative to the bridge axis. If there is a displacement of 15 centimeters, the maximum bending moment in the beam, to the side which the shift occurred, is equal to $775 \mathrm{kN} \cdot \mathrm{m}$, i.e. the increase in internal force (bending moment) is $8.38 \%$.

2) When the axis of the track panel is shifted by the above mentioned value, an additional $35 \mathrm{kN} \cdot \mathrm{m}$ torque appears.

3) The maximum stress in the compressed zone is $3.91 \mathrm{MPa}$, and before the axis of the track panel is displaced relative to the bridge axis, this value was $3.69 \mathrm{MPa}$.

4) The maximum stress in the stretched zone is $6.22 \mathrm{MPa}$, this value was $5.85 \mathrm{MPa}$ before displacement, thus, the bending stress increases by $5.95 \%$.

5) An additional stress of $0.28 \mathrm{MPa}$ appears due to the additional torsion of the span beam.

6) Despite the increase in stresses generally can be considered insignificant, the presence of defects in the beams of the span in the form of concrete chips, a stretched zone, exposure and corrosion of the principle reinforcement, a decrease in the calculated cross section, leaching of cement stone from the concrete of the beams, a decrease in the strength of concrete over time, can make an increase in design stresses of almost $6 \%$ a very significant factor limiting the operational capabilities of bridge crossings.

\section{References}

1. A. Loktev, V. Korolev, I. Shishkina et al. TransSiberia 2019: VIII International Scientific Siberian Transport Forum, 2, 1116 (2020) DOI: 10.1007/978-3-030-37919-3

2. A. Loktev, V. Korolev et al. IOP Conf. Series: Materials Science and Engineering, 463, 032019 (2018) DOI: 10.1088/1757-899X/463/3/032019

3. A. Loktev, V. Korolev et al. Vestnik of the Railway Research Institute, 77(6), 331-336 (2018) DOI: 10.21780/2223-9731-2018-77-6-331-336 
4. V. Korolev. TransSiberia 2019: VIII International Scientific Siberian Transport Forum, 175-187 DOI: 10.1007/978-3-030-37919-3_17

5. A.V. Savin, V.V. Korolev, I.V. Shishkina. IOP Conf. Series: Materials Science and Engineering, 687, 022035 (2019) DOI: 10.1088/1757-899X/687/2/022035

6. A. Savin, V. Korolev et al. TransSiberia 2019: VIII International Scientific Siberian Transport Forum, 797-808 DOI: 10.1007/978-3-030-37916-2_78

7. A. Loktev, V. Korolev et al. IOP Conf. Series: Materials Science and Engineering, 463, 032018 (2018) DOI: 10.1088/1757-899X/463/3/032018

8. D.A. Gura, I.G. Markovskii, B.A. Hahuk, S.K. Pshidatok, IOP Conference Series: Materials Science and Engineering 698(4), 044014 (2019). DOI: 10.1088/1757899X/698/4/044014

9. A. Loktev, V. Korolev et al. Procedia Engineering, 189, 133-137 (2017) DOI: 10.1016/j.proeng.2017.05.022

10. A. Savin, A. Kogan et al. International Journal of Innovative Technology and Exploring Engineering, 8(7), 2325-2328 (2019)

11. A. Loktev, V. Korolev et al. Advances in Intelligent Systems and Computing, 982, 325-336 (2020) DOI: 10.1007/978-3-030-19756-8_30

12. A. Savin, O. Suslov et al. TransSiberia 2019: VIII International Scientific Siberian Transport Forum, 648-654 DOI: 10.1007/978-3-030-37916-2_62

13. B. Glusberg, A. Loktev et al. Advances in Intelligent Systems and Computing, 982, 337-345 (2020) DOI: 10.1007/978-3-030-19756-8_31

14. A. Lyudagovsky, A. Loktev, V. Korolev et al. E3S Web of Conferences, 110, 01017 (2019) DOI: $10.1051 /$ e3sconf $/ 201911001017$

15. S. Ognjenovic, R. Donceva, N. Vatin. Procedia Engineering. 2015. 117(1). Pp. 544550. DOI:10.1016/j.proeng.2015.08.210.

16. B. Glusberg, A. Savin et al. Advances in Intelligent Systems and Computing, 982, 571587 (2020) DOI: 10.1007/978-3-030-19756-8_54

17. B. Glusberg, V. Korolev et al. MATEC Web of Conferences, 239, 01054 (2018) DOI: $10.1051 /$ matecconf $/ 201823901054$

18. B. Glusberg, V. Korolev et al. E3S Web of Conferences, 138, 01017 (2019) DOI: $10.1051 / \mathrm{e} 3$ sconf $/ 201913801017$

19. A.A. Loktev, V.V. Korolev, E.A. Gridasova. IOP Conf. Series: Materials Science and Engineering, 687, 022036 (2019) DOI: 10.1088/1757-899X/687/2/022036

20. V. Korolev. TransSiberia 2019: VIII International Scientific Siberian Transport Forum, 621-638 DOI: 10.1007/978-3-030-37916-2_60

21. V. Korolev, A. Loktev et al. IOP Conference Series: Earth and Environmental Science, 403, DOI: 10.1088/1755-1315/403/1/012194

22. E. Gridasova, P. Nikiforov, A. Loktev et al. TransSiberia 2019: VIII International Scientific Siberian Transport Forum, 559-569 DOI: 10.1007/978-3-030-37916-2_54

23. I. Shishkina. TransSiberia 2019: VIII International Scientific Siberian Transport Forum, 834-844, DOI: 10.1007/978-3-030-37916-2_82 